\title{
Redundant variables and Granger causality
}

\author{
L. Angelini, ${ }^{1,2}$ M. de Tommaso, ${ }^{3}$ D. Marinazzo, ${ }^{4}$ L. Nitti,,${ }^{1,5}$ M. Pellicoro, ${ }^{1,2}$ and S. Stramaglia ${ }^{1,2}$ \\ ${ }^{1}$ Istituto Nazionale di Fisica Nucleare, Sezione di Bari, Bari, Italy \\ ${ }^{2}$ Dipartimento di Fisica, University of Bari, Bari, Italy \\ ${ }^{3}$ Dipartimento di Scienze Neurologiche e Psichiatriche, University of Bari, Bari, Italy \\ ${ }^{4}$ Laboratoire de Neurophysique et Physiologie, CNRS UMR 8119, Université Paris Descartes, Paris, France \\ ${ }^{5}$ Dipartimento di Biochimica Medica, Biologia Medica e Fisica Medica, University of Bari, Bari, Italy \\ (Received 28 September 2009; revised manuscript received 28 December 2009; published 5 March 2010)
}

\begin{abstract}
We discuss the use of multivariate Granger causality in presence of redundant variables: the application of the standard analysis, in this case, leads to under estimation of causalities. Using the un-normalized version of the causality index, we quantitatively develop the notions of redundancy and synergy in the frame of causality and propose two approaches to group redundant variables: (i) for a given target, the remaining variables are grouped so as to maximize the total causality and (ii) the whole set of variables is partitioned to maximize the sum of the causalities between subsets. We show the application to a real neurological experiment, aiming to a deeper understanding of the physiological basis of abnormal neuronal oscillations in the migraine brain. The outcome by our approach reveals the change in the informational pattern due to repetitive transcranial magnetic stimulations.
\end{abstract}

DOI: 10.1103/PhysRevE.81.037201

PACS number(s): 05.45.Tp, 87.19.L-

Wiener [1] and Granger [2] formalized the notion that if the prediction of one time series could be improved by incorporating the knowledge of past values of a second one, then the latter is said to have a causal influence on the former. Initially developed for econometric applications, Granger causality has gained popularity also among physicists (see, e.g., [3-7]). A kernel method for Granger causality, introduced in [8], deals with the nonlinear case by embedding data onto an Hilbert space, and searching for linear relations in that space. Geweke [9] has generalized Granger causality to a multivariate fashion in order to identify conditional Granger causality; as described in [10], multivariate causality may be used to infer the structure of dynamical networks [11] from data.

Granger causality is connected to the information flow between variables [12]. Another important notion in information theory is the redundancy in a group of variables, formalized in [13] as a generalization of the mutual information. A formalism to recognize redundant and synergetic variables in neuronal ensembles has been proposed in [14] and generalized in [15]; the information theoretic treatments of groups of correlated degrees of freedom can reveal their functional roles in complex systems.

The purpose of this work is to show that the presence of redundant variables influences the performance by multivariate Granger causality and to propose a novel approach to exploit redundancy so as to identify functional patterns in data. In the following, we provide a quantitative definition to recognize redundancy and synergy in the frame of causality and show that the maximization of the total causality is connected to the detection of groups of redundant variables.

Let us consider $n$ time series $\left\{x_{\alpha}(t)\right\}_{\alpha=1, \ldots, n}[16]$; the state vectors are denoted

$$
X_{\alpha}(t)=\left[x_{\alpha}(t-m), \ldots, x_{\alpha}(t-1)\right],
$$

$m$ being the window length (the choice of $m$ can be done using the standard cross-validation scheme). Let $\epsilon\left(x_{\alpha} \mid \mathbf{X}\right)$ be the mean squared error prediction of $x_{\alpha}$ on the basis of all the vectors $\mathbf{X}$ (corresponding to linear regression or non linear regression by the kernel approach described in [8]). The multivariate Granger causality index $\delta(\beta \rightarrow \alpha)$ is defined as follows: consider the prediction of $x_{\alpha}$ on the basis of all the variables but $X_{\beta}$ and the prediction of $x_{\alpha}$ using all the variables, then the causality is the (normalized) variation of the error in the two conditions, i.e.,

$$
\delta(\beta \rightarrow \alpha)=\frac{\epsilon\left(x_{\alpha} \mid \mathbf{X} \backslash X_{\beta}\right)-\epsilon\left(x_{\alpha} \mid \mathbf{X}\right)}{\epsilon\left(x_{\alpha} \mid \mathbf{X} \backslash X_{\beta}\right)} .
$$

Here we use the selection of significative eigenvalues described in [8] to address the problem of over-fitting in Eq. (1).

The straightforward generalization of Granger causality for sets of variables is

$$
\delta(B \rightarrow A)=\sum_{\alpha \in A} \frac{\epsilon\left(x_{\alpha} \mid \mathbf{X} \backslash B\right)-\epsilon\left(x_{\alpha} \mid \mathbf{X}\right)}{\epsilon\left(x_{\alpha} \mid \mathbf{X} \backslash B\right)},
$$

where $A$ and $B$ are two disjoint subsets of $\{1, \ldots, n\}$, and $\mathbf{X} \backslash B$ means the set of all variables except for those $X_{\beta}$ with $\beta \in B$.

On the other hand, the un-normalized version of it, i.e.,

$$
\delta^{u}(B \rightarrow A)=\sum_{\alpha \in A}\left\{\boldsymbol{\epsilon}\left(x_{\alpha} \mid \mathbf{X} \backslash B\right)-\epsilon\left(x_{\alpha} \mid \mathbf{X}\right)\right\},
$$

can be easily be shown to satisfy the following interesting property: if $\left\{X_{\beta}\right\}_{\beta \in B}$ are statistically independent and their contributions in the model for $\mathrm{A}$ are additive, then

$$
\delta^{u}(B \rightarrow A)=\sum_{\beta \in B} \delta^{u}(\beta \rightarrow A) .
$$

In order to identify the informational character of a set of variables $B$, concerning the causal relationship $B \rightarrow A$, we remind that, in general, synergy occurs if $B$ contributes to $A$ with more information than the sum of all its variables, while 
redundancy corresponds to situations with the same information being shared by the variables in $B$. Following [13-15], we make quantitative these notions and define the variables in $B$ redundant if $\delta^{u}(B \rightarrow A)>\sum_{\beta \in B} \delta^{u}(\beta \rightarrow A)$, and synergetic if $\delta^{u}(B \rightarrow A)<\Sigma_{\beta \in B} \delta^{u}(\beta \rightarrow A)$. In order to justify these definitions, first we observe that the case of independent variables (and additive contributions) does not fall in the redundancy case neither in the synergetic case, due to Eq. (4), as it should be. Moreover, we describe the following example for two variables $X_{1}$ and $X_{2}$. If $X_{1}$ and $X_{2}$ are redundant, then removing $X_{1}$ from the input variables of the regression model does not have a great effect, as $X_{2}$ provides the same information as $X_{1}$; this implies that $\delta^{u}\left(X_{1} \rightarrow A\right)$ is nearly zero. The same reasoning holds for $X_{2}$, hence we expect that $\delta^{u}\left[\left(X_{1}, X_{2}\right) \rightarrow A\right]>\delta^{u}\left(X_{1} \rightarrow A\right)+\delta^{u}\left(X_{2} \rightarrow A\right)$. Conversely, let us suppose that $X_{1}$ and $X_{2}$ are synergetic, i.e., they provide some information about $A$ only when both the variables are used in the regression model; in this case $\delta^{u}\left(\left\{X_{1}, X_{2}\right\} \rightarrow A\right), \quad \delta^{u}\left(X_{1} \rightarrow A\right)$ and $\delta^{u}\left(X_{2} \rightarrow A\right)$ are almost equal and, therefore, $\delta^{u}\left(\left\{X_{1}, X_{2}\right\} \rightarrow A\right)<\delta^{u}\left(X_{1} \rightarrow A\right)+\delta^{u}\left(X_{2}\right.$ $\rightarrow A)$.

Two analytically tractable cases are now reported as examples. Consider two stationary and Gaussian time series $x(t)$ and $y(t)$ with $\left\langle x^{2}(t)\right\rangle=\left\langle y^{2}(t)\right\rangle=1$ and $\langle x(t) y(t)\rangle=\mathcal{C}$; they correspond, e.g., to the asymptotic regime of the autoregressive system,

$$
\begin{aligned}
& x_{t+1}=a x_{t}+b y_{t}+\sigma \xi_{t+1}^{(1)}, \\
& y_{t+1}=b x_{t}+a y_{t}+\sigma \xi_{t+1}^{(2)},
\end{aligned}
$$

where $\xi$ are independent and identically distributed unit variance Gaussian variables, $\mathcal{C}=2 a b /\left(1-a^{2}-b^{2}\right)$ and $\sigma^{2}=1-a^{2}$ $-b^{2}-2 a b C$. Considering the time series $z_{t+1}=A\left(x_{t}+y_{t}\right)$ $+\sigma^{\prime} \xi_{t+1}^{(3)}$ with $\sigma^{\prime}=\sqrt{1-2 A^{2}(1+\mathcal{C})}$, we obtain for $m=1$,

$$
\delta^{u}(\{x, y\} \rightarrow z)-\delta^{u}(x \rightarrow z)-\delta^{u}(y \rightarrow z)=A^{2}\left(\mathcal{C}+\mathcal{C}^{2}\right)
$$

Hence, $x$ and $y$ are redundant (synergetic) for $z$ if $\mathcal{C}$ is positive (negative). Turning to consider $w_{t+1}=B x_{t} \cdot y_{t}+\sigma^{\prime \prime} \xi_{t+1}^{(4)}$ with $\sigma^{\prime \prime}=\sqrt{1-B^{2}(1+2 \mathcal{C})^{2}}$, and using the polynomial kernel with $p=2$, we have,

$$
\delta^{u}(\{x, y\} \rightarrow z)-\delta^{u}(x \rightarrow z)-\delta^{u}(y \rightarrow z)=B^{2}\left(4 \mathcal{C}^{2}-1\right)
$$

$x$ and $y$ are synergetic (redundant) for $w$ if $|\mathcal{C}|<0.5$ $(|\mathcal{C}|>0.5)$.

The presence of redundant variables leads to under estimation of their causality when the standard multivariate approach is applied (this is not the case for synergetic variables). Redundant variables should be grouped to get a reliable measure of causality, and to characterize interactions in a more compact way. As it is clear from the discussion above, grouping redundant variables is connected to maximization of the un-normalized causality index [Eq. (3)] and, in the general setting, can be made as follows. For a given target $\alpha_{0}$, we call $B$ the set of the remaining $n-1$ variables. The partition $\left\{A_{\ell}\right\}$ of $B$, maximizing the total causality,

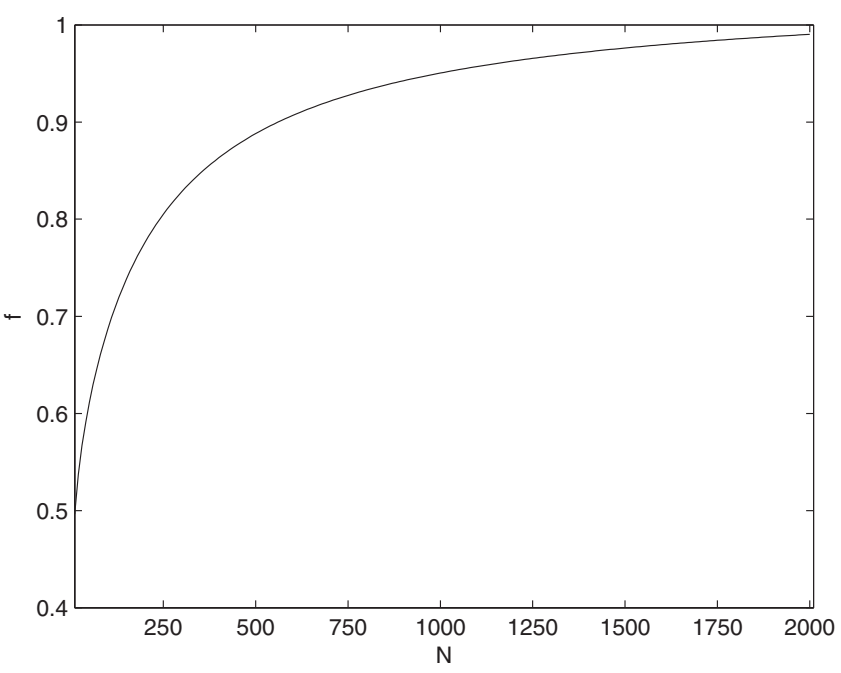

FIG. 1. The fraction $f$ of times that the $x$ and $y$ are recognized as redundant for the variable $z$ (see the text), versus the number of samples $N . f$ is evaluated over $10^{6}$ repetitions.

$$
\Delta=\sum_{\ell} \delta^{u}\left(A_{\ell} \rightarrow x_{\alpha_{0}}\right)
$$

consists of groups of redundant variables. Concerning the problem of finite sample size, we consider $N$ samples from Eq. (5), with $a=0.5$ and $b=0.4$, and estimate casualties on these data. In Fig. 1, we depict, as a function of $N$, the fraction $f$ of times that the $x$ and $y$ are recognized as redundant for the variable $z$ (with $A=0.4$ ); a large amount of data is needed to assess significative causality and so to discover redundancy. The present approach can, thus, be used only in applications such that a large number of samples is available.

Another example consists of a system of nine oscillators evolving according to noisy Kuramoto's equations [17],

$$
\dot{\theta}_{i}=\omega_{i}+K \sum_{j=1}^{9} \sin \left(\theta_{j}-\theta_{i}\right)+\xi_{i}(t) ;
$$

We consider three groups of oscillators, each made of three oscillators with the same natural frequency, respectively $\omega$ $=1,2,4$; the noise strength is 0.01 . Using the approach for circular variables, described in [18], we find that the partition $\left\{A_{\ell}\right\}$ of the nine oscillators, maximizing the sum of the causalities between every pair of subsets,

$$
\Gamma=\sum_{\ell} \sum_{\ell^{\prime} \neq \ell} \delta^{u}\left(A_{\ell} \rightarrow A_{\ell^{\prime}}\right),
$$

is $\{1,2,3\}\{4,5,6\}\{7,8,9\}$, corresponding to oscillators with the same natural frequency belonging to the same subset. In Fig. 2 , we depict the optimal $\Gamma$ and the value of $\Gamma$ corresponding to the partition where each oscillator constitutes a set, versus the coupling $K$. It is clear that the maximization of $\Gamma$ reveals the structure of the system in this example.

Now, we turn to consider a real application, i.e., electroencephalographic data from nineteen subjects suffering from migraine, under steady state flash stimuli $(9 \mathrm{~Hz})$ and repetitive transcranial magnetic stimulation (rTMS), a noninvasive method to excite neurons in the brain [19]. Migraine is a 


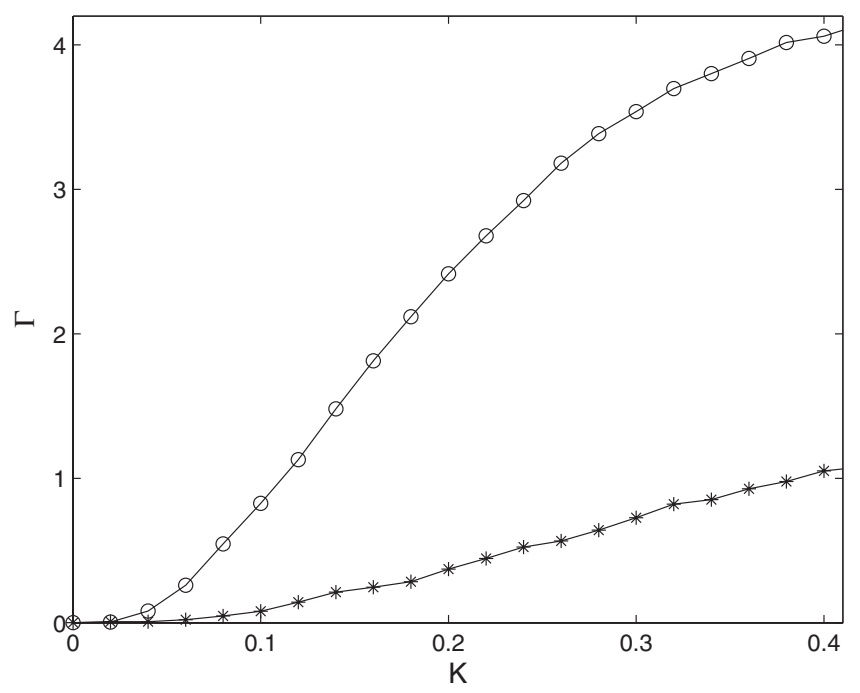

FIG. 2. Concerning the system of nine oscillators described in the next, we depict the sum of the causalities between every pair of subsets $\Gamma$ (see the text) corresponding to the partitions $\{1,2,3\}\{4,5,6\}\{7,8,9\}$ (empty circles) and $\{1\}\{2\}\{3\}\{5\}\{6\}\{7\}\{8\}\{9\}$ (stars). Causalities are estimated over 5000 samples for each value of $K$.

complex disorder of neurovascular origin whose pathophysiological basis is largely unknown. An altered cortical excitability may activate the trigemino-vascular system, but the question about a basal hypo or hyper cortical excitability is actually a matter of debate [20]. In a previous work [21], anomalous cortical synchronization in migraneurs under flash stimuli has been reported. A better understanding of migraine pathophysiology may improve its therapeutical approach: in this view, studies employing neurophysiological techniques, possibly supported by advanced methods of quantitative analysis, may give an aid to the knowledge of migraine pathophysiology [22]. An important feature of migraine brain, is the tendency to hypersynchronization of alpha rhythms, which is influenced by antiepileptic drugs [23].
rTMS induces a cortical modulation that lasts beyond the time of stimulation [24]: its effects depend on the frequency of stimulations. In order to understand the physiological basis of abnormal neuronal oscillations in migraine brain, we apply $1 \mathrm{~Hz}$ rTMS over the occipital cortex, before performing repetitive flash stimulation. The records are $12 \mathrm{~s}$ long, sampled at $256 \mathrm{~Hz}$ : this EEG duration is representative of the pattern of brain responsiveness to light stimuli, as previously shown [21]. The signals are measured on seven channels (Fz,P3,P4,Cz,O1,Oz,O2) in three conditions basal (only flash stimuli) sham (placebo, i.e., flash stimuli and a fake magnetic stimulator) and rTMS (flash stimuli and magnetic stimulations). As in the example above, for each target channel we exhaustively search for the partition of the remaining six channels, which leads to the highest total causality $\Delta$ (averaged over the nineteen patients). In basal and sham conditions, we find that, for each target channel, the optimal partition is always a single set containing all the six remaining channels, in other words all the channels are redundant in these conditions. In presence of rTMS the causality pattern becomes more complex, and not all sets of variables are redundant with respect to the prediction of the others. All the six remaining channels are redundant for targets Fz,P4,O1,Oz; for the other channels the best partitions are

$$
\begin{aligned}
& \{F z, P 4, O 1, O z, O 2\}\{P 3\} \rightarrow C z, \\
& \{F z, P 4, O 1, O z, O 2\}\{C z\} \rightarrow P 3, \\
& \{F z, P 4, O 1, O z\}\{C z P 3\} \rightarrow O 2 .
\end{aligned}
$$

These relations suggest the presence of a new source of information, due to magnetic stimulations, corresponding to $\mathrm{Cz}$ and P3 channels. We also search for the partition of the seven channel maximizing the total causality between groups $(\Gamma)$, averaged over the patients. We find that the best partition is $\{F z, P 4, O 1, O 2\}\{C z P 3 O z\}$ for basal and sham conditions. For the TMS condition, instead, the best partition is $\{F z, P 4, O z\}\{C z P 3\}\{O 1\}\{O 2\}$; this result is consistent with

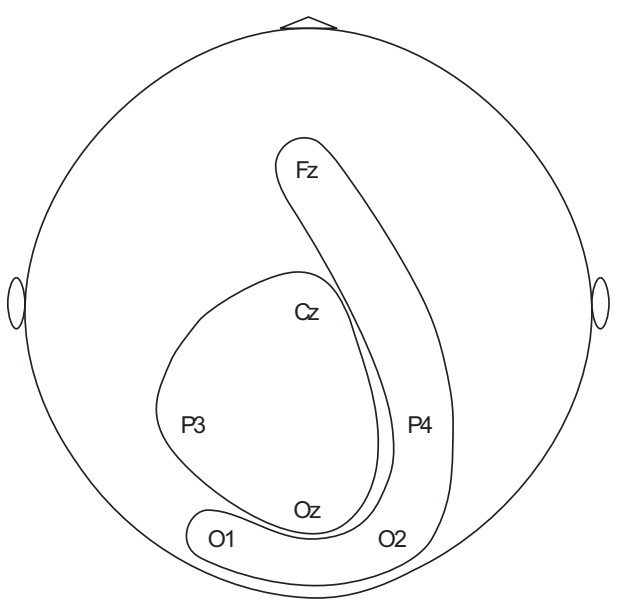

a)

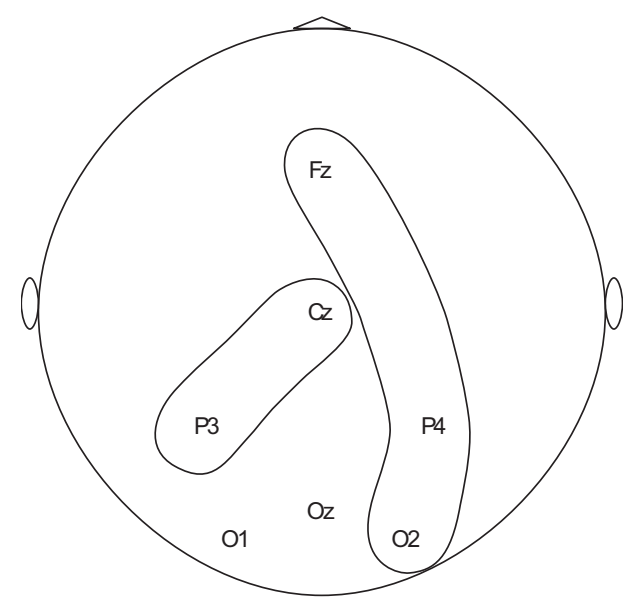

b)

FIG. 3. The partitions of electrodes maximizing $\Gamma$ (see the text). Left: the optimal partition for Basal and Sham conditions. Right: the optimal partition in presence of TMS. 
the previous analysis as the channels $\mathrm{Cz}$ and P3 are grouped, see Fig. 3.

The change of the informational pattern, induced by occipital cortex inhibition, may confirm that neuronal oscillations are related to the state cortical excitability. Presently, we have no explanation about the significance of the specific $\mathrm{Cz}-\mathrm{P} 3$ group related to rTMS effect, but we can assert that oscillations in migraine brain vary as a function of cortical excitability. The reliability of this pattern in migraine needs to be matched with a control group, so as to better understand the peculiar reactivity of migraine brain and to find the optimal way to influence it. Some remarks are in order. Averaging over patients is mandatory to reduce the effects due to the variability among subjects. Our results are obtained using the linear kernel and $m=1$, but the same partitions are obtained using the quadratic kernel and $m=2$ (application of cross validation, on these data, suggests a low value of the order $\mathrm{m}$; therefore we restrict our analysis to $m=1$ and 2).
We find, in this real application, that the optimal partition maximizing the total causality is unique in all cases. It may happen, in other instances, that several partitions have the same total causality: in those cases prior information should be used to select one of the degenerate partitions.

Summarizing, in this work we have quantitatively developed the notions of redundancy and synergy in the frame of causality. We have proposed to generalize the standard multivariate Granger method in presence of redundant variables, by using the causality index without normalization, and analyzing the system as follows: (i) for a given target, the remaining variables are grouped so as to maximize the total causality and (ii) the whole set of variables is partitioned to maximize the sum of the causalities between groups. Analyzing real data from a neurophysiological experiments, the proposed approach was able to detect the informational pattern induced by magnetic stimulations.
[1] N. Wiener, in Modern Mathematics for Engineers, edited by E. F. Beckenbach (McGraw-Hill, New York, 1956).

[2] C. W. J. Granger, Econometrica 37, 424 (1969).

[3] Y. Chen, G. Rangarajan, J. Feng, and M. Ding, Phys. Lett. A 324, 26 (2004).

[4] K. J. Blinowska, R. Kus, and M. Kaminski, Phys. Rev. E 70, 050902(R) (2004).

[5] D. A. Smirnov and B. P. Bezruchko, Phys. Rev. E 79, 046204 (2009); D. A. Smirnov and I. I. Mokhov, ibid. 80, 016208 (2009).

[6] M. Dhamala, G. Rangarajan, and M. Ding, Phys. Rev. Lett. 100, 018701 (2008).

[7] K. Ishiguro, N. Otsu, M. Lungarella, and Y. Kuniyoshi, Phys. Rev. E 77, 036217 (2008).

[8] D. Marinazzo, M. Pellicoro, and S. Stramaglia, Phys. Rev. Lett. 100, 144103 (2008).

[9] J. Geweke, J. Am. Stat. Assoc. 79, 907 (1984).

[10] D. Marinazzo, M. Pellicoro, and S. Stramaglia, Phys. Rev. E 77, 056215 (2008).

[11] S. Boccaletti, V. Latora, Y. Moreno, M. Chavez, and D. U. Hwang, Phys. Rep. 424, 175 (2006).

[12] K. Hlavackova-Schindler, M. Palus, M. Vejmelka, and J. Bhat- tacharya, Phys. Rep. 441, 1 (2007).

[13] M. Palus, V. Albrecht, and I. Dvorak, Phys. Lett. A 175, 203 (1993).

[14] E. Schneidman, W. Bialek, and M. J. Berry, J. Neurosci. 23, 11539 (2003).

[15] L. M. A. Bettencourt, V. Gintautas, and M. I. Ham, Phys. Rev. Lett. 100, 238701 (2008).

[16] After a linear transformation, we may assume all the time series to have zero mean and unit variance.

[17] Y. Kuramoto, Chemical Oscillations, Waves and Turbulence (Springer, Berlin, 1984).

[18] L. Angelini, M. Pellicoro, and S. Stramaglia, Phys. Lett. A 373, 2467 (2009).

[19] A. T. Barker, R. Jalinous, and I. L. Freeston, Lancet 325, 1106 (1985)

[20] G. Coppola, F. Pierelli, and J. Schoenen, Cephalalgia 27, 1427 (2007).

[21] L. Angelini et al., Phys. Rev. Lett. 93, 038103 (2004).

[22] M. Valeriani, Clin. Neurophysiol. 116, 2717 (2005).

[23] M. de Tommaso et al., Clin. Neurophysiol. 118, 2297 (2007).

[24] F. Fregni et al., Lancet Neurol. 6, 188 (2007). 